\title{
FINITE GROUPS \\ WITH A NILPOTENT MAXIMAL SUBGROUP
}

\author{
ZVONIMIR JANKO \\ (received 8 January 1964)
}

Let $G$ be a finite group all of whose proper subgroups are nilpotent. Then by a theorem of Schmidt-Iwasawa the group $G$ is soluble. But what can we say about a finite group $G$ if only one maximal subgroup is nilpotent?

Let $G$ be a finite group with a nilpotent maximal subgroup $M$. Then' the following results are known:

Theorem of J. G. Thompson [4]. If $M$ has odd order, then $G$ is soluble.

TheOREM of Deskins [1]. If $M$ has class $\leqq 2$, then $G$ is soluble.

TheOREM of JANKo [3]. If the 2-Sylow subgroup $M_{2}$ of $M$ is abelian, then $G$ is soluble.

Now we can give a very simple proof of the following

THEOREM. Let $G$ be a finite group with a nilpotent maximal subgroup $M$. If a 2-Sylow subgroup $M_{2}$ of $M$ has class $\leqq 2$, then $G$ is soluble.

This result is the best possible because the simple group $\operatorname{LF}(2,17)$ has a 2-Sylow subgroup of class 3 which is a maximal subgroup.

The theorem was announced without proof in [3]. The proof of the theorem will be independent of the theorem of Deskins. We shall give at first some definitions.

Definition 1. A finite group $G$ is called p-nilpotent if it has a normal Sylow $p$-complement.

Definition 2. Let $Z\left(G_{p}\right)$ be the centre of a $p$-Sylow subgroup $G_{p}$ of a finite group $G$. Then the group $G$ is called p-normal if $Z\left(G_{p}\right)$ is the centre of every $p$-Sylow subgroup of $G$ in which it is contained.

Definition 3. Let $\alpha$ be an automorphism of a group $G$. Then $\alpha$ is called fixed-point-free if and only if $\alpha$ fixes only the unit element of $G$.

In the proof of the theorem we shall use the theorem of J. G. Thompson and also the following two results:

TheOREM OF GRÜN-WIElandT-P. Hall [2]. Let $G$ be a finite p-normal group and $Z\left(G_{p}\right)$ the centre of a $p$-Sylow subgroup $G_{p}$ of $G$. Then $G$ is $p$ nilpotent if and only if the normalizer $N\left(Z\left(G_{p}\right)\right)$ of $Z\left(G_{p}\right)$ is p-nilpotent. 
Theorem of ZassenhaUs [5]. If a finite group $G$ has a fixed-point-free automorphism of order 2, then $G$ is an abelian group.

PROOF OF THE THEOREM. Using induction on the order we can assume that there is no non-trivial normal subgroup of $G$ contained in $M$. If $M_{p}$ is a $p$-Sylow subgroup of $M$, then $M_{p}$ is normal in $M$ and $M_{p}$ must be a $p$-Sylow subgroup of $G$. Hence $M$ is a Hall subgroup of $G$.

Suppose now that $M$ is not a Sylow subgroup of $G$. In this case we can prove that $G$ is $p$-normal for every prime $p$ which divides the order of $M$. Let $Z=Z\left(M_{p}\right)$ be the centre of the $p$-Sylow subgroup $M_{p}$ of $M$. Suppose that

$$
Z \leqq M_{p}^{x}=x^{-1} M_{p} x
$$

for $a$ certain $x \in G$. Then

$$
C(Z) \geqq\left\{M,\left(M^{*}\right)_{D^{\prime}}\right\}=M,
$$

where $C(Z)$ denotes the centralizer of $Z$ and $1 \neq\left(M^{x}\right)_{p}$, is a $p$-Sylow complement of $M^{x}$. Consequently $M_{p^{\prime}}^{x}=M_{p^{\prime}}$ and $x \in N\left(M_{p^{\prime}}\right)$. On the other hand $N\left(M_{p^{\prime}}\right)=M$ whence $x \in M$. So $Z$ is the centre of $\left(M_{p}\right)^{x}=M_{p}$ and the group $G$ is $p$-normal. By the theorem of Grün-Wielandt-P. Hall the group $G$ is $p$-nilpotent for every prime $p$ which divides the order $|M|$ of $M$. Let $N_{p}$ denote a normal $p$-Sylow complement of $G$. Then we consider the intersection

$$
N=\bigcap_{p|| M \mid} N_{p}
$$

The group $N$ is obviously a normal complement of $M$.

By the theorem of J. G. Thompson we can suppose that $M$ has even order. Let $\tau$ be a central involution of $M$. Because $C(\tau)=M$ the involution $\tau$ acts fixed-point-free on $N$ and by the theorem of Zassenhaus $N$ is abelian and so $G$ is soluble.

We suppose now that $M$ is a Sylow subgroup of $G$. By the theorem of J. G. Thompson and by our assumption we can suppose that $M$ is a 2-Sylow subgroup of class $\leqq 2$.

If $G$ is 2-normal (and because $N(Z(M))=M$ ), then $G$ is 2-nilpotent by the theorem of Grün-Wielandt-P. Hall. Let $N$ be a normal 2-complement of $G$. Then again by the theorem of Zassenhaus $N$ is abelian and $G$ is soluble.

If $G$ is not 2-normal, then there is an $x \in G$ such that $Z(M) \leqq M \cap M^{x}=D$ and $M^{x} \neq M$. But then (because $M^{\prime} \leqq Z(M)$ ) $D$ is normal in $M$ and $N(D) \cap M^{x} \neq D$ which gives $N(D)=G$. But this is impossible because we have assumed that $M$ does not contain non-trivial normal subgroups of $G$. The proof is complete. 


\section{References}

[1] Deskins, W. E., A condition for the solvability of a finite group, Illinois J. Math. 5 (1961), $306-313$.

[2] Hall, M., The theory of groups. New York 1959.

[3] Janko, Z., Verallgemeinerung eines Satzes von B. Huppert und J. G. Thompson, Arch. Math. 12 (1961), 280-281.

[4] Thompson, J. G., Normal p-complements for finite groups, Math. Z. 72 (1960), $332-354$.

[5] Zassenhaus, H., Kennzeichnung endlicher linearen Gruppen als Permutationsgruppen, Abh. Hamburg Math. Sem. 11 (1936), 17-40.

Department of Mathematics, Australian National University, Canberra. 